M/C-TH 99-16

DAMTP-1999-167

\title{
EXCLUSIVE VECTOR PHOTOPRODUCTION: CONFIRMATION OF REGGE THEORY
}

\author{
A Donnachie \\ Department of Physics, Manchester University \\ P V Landshoff \\ DAMTP, Cambridge University*
}

\begin{abstract}
Recent small- $t$ ZEUS data for exclusive $\rho$ photoproduction are in excellent agreement with exchange of the classical soft pomeron with slope $\alpha^{\prime}=0.25 \mathrm{GeV}^{-2}$. Adding in a flavour-blind hard-pomeron contribution, whose magnitude is calculated from the data for exclusive $J / \psi$ photoproduction, gives a good fit also to the ZEUS data for $\rho$ photoproduction at larger values of $t$, and to $\phi$ photoproduction.
\end{abstract}

The ZEUS collaboration has recently suggested ${ }^{[1]}$ that their data for exclusive $\rho$ photoproduction at HERA, when combined with lower-energy data ${ }^{[2]}$ lead to a slope $\alpha^{\prime}$ for the trajectory of the soft pomeron that differs significantly from the classical value $\alpha^{\prime}=0.25 \mathrm{GeV}^{-2}$. A main message of this paper is to disagree with this conclusion; we show that in fact the classical value is confirmed by the data.

The slope $\alpha^{\prime}$ should be determined from the data at small $t$, but the ZEUS measurements extend also to rather large $t$. At HERA energy, soft-pomeron exchange dominates the differential crosssection out to values of $|t|$ of about $0.4 \mathrm{GeV}^{2}$. Beyond that, some new contribution is needed. For exclusive $J / \psi$ photoproduction, a new contribution is needed even at very small $t$. We have shown recently $[3]$ that introducing a "hard pomeron" gives an excellent description of data not only for $J / \psi$ photoproduction, but also for the charm structure function $F_{2}^{c}$ and the small- $x$ behaviour of the total structure function $F_{2}$. A second message in the present paper is that the introduction of the same hard pomeron also provides a good description of the large- $t \rho$ photoproduction data. Applying the model to $\phi$ photoproduction gives a satisfactory description of these data too.

Consider first small- $t \rho$ photoproduction. In order to extract the soft-pomeron slope $\alpha^{\prime}$, it is necessary to consider data from HERA together with measurements at much lower energy. We have shown previously ${ }^{[4]}$ that the description of the lower-energy data needs a significant contribution from $f$ and $a_{2}$ exchange; this is missing from the ZEUS analysis ${ }^{[1]}$. Further, as is apparent from the data shown in figure 1, the relative normalisation of the lower energy experiments is somewhat erratic and it is not correct to use just one or two energies for comparison. It is necessary to perform a global fit to average out the discrepancies. In our previous analysis ${ }^{[4]}$, we first assumed that the contribution from soft-pomeron and $f, a_{2}$ exchanges to the $\rho^{0} p$ total cross section is the same as in the average of the $\pi^{+} p$ and $\pi^{-} p$ cross sections ${ }^{[5]}$. We then used $\rho$-dominance, with a factor of 0.84 to allow for finite-width corrections to $\rho \rightarrow e^{+} e^{-}$decay ${ }^{[6]}$, to calculate the forward differential cross section for $\gamma p \rightarrow \rho p$. Figure 1 (solid curve) shows the resulting cross section at $t=0$ as a function of energy.

* email addresses: ad@a3.ph.man.ac.uk, pvl@damtp.cam.ac.uk 


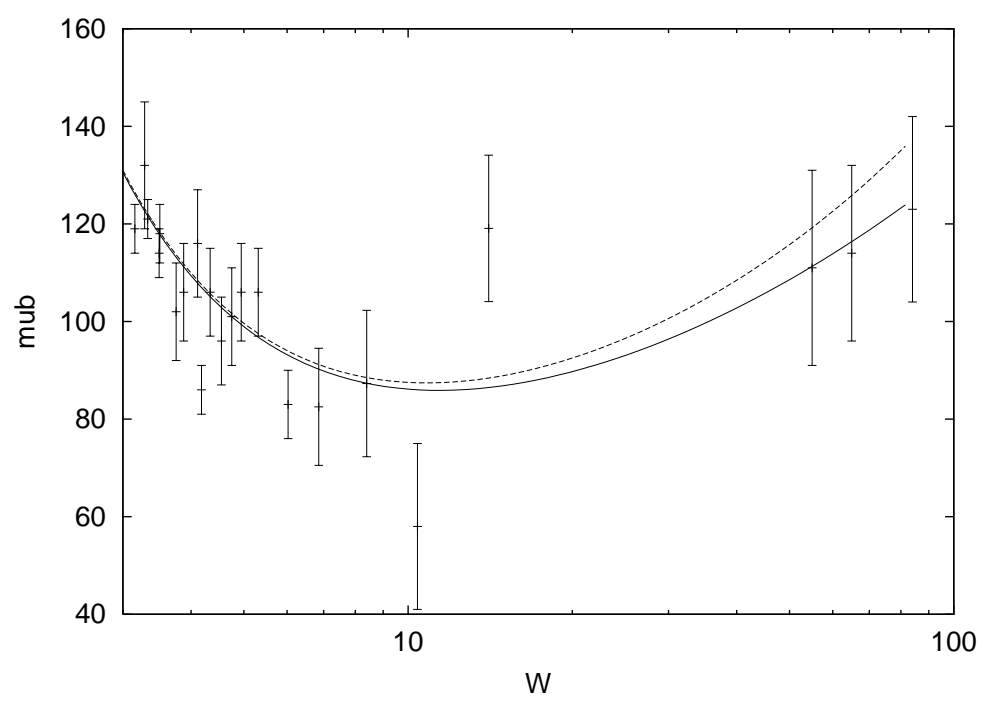

Figure 1: Data for the forward differential cross section for exclusive $\rho^{0}$ photoproduction. The solid curve corresponds to the exchange of the soft pomeron together with $f$ and $a_{2}$, while the dashed curve includes also a hard-pomeron contribution.

In order to extend this away from the forward direction, we need the two Regge trajectories

$$
\begin{aligned}
\alpha_{P_{1}}(t) & =1.08+\alpha_{P_{1}}^{\prime} t & \alpha_{P_{1}}^{\prime}=0.25 \\
\alpha_{R}(t) & =0.55+\alpha_{R}^{\prime} t & \alpha_{R}^{\prime}=0.93
\end{aligned}
$$

We need also to decide the mass scale $s_{0}$ by which we must divide $s$ before we raise it to the Regge power. There is no theory that determines this. We adopt the dual-model prescription ${ }^{[7]}$ that, for a trajectory of slope $\alpha^{\prime}$, one should take $s_{0}=1 / \alpha^{\prime}$. It is well-established ${ }^{[8]}$ that the trajectories couple to the proton through the Dirac electric form factor

$$
F(t)=\frac{4 m^{2}-2.79 t}{4 m^{2}-t}\left(\frac{1}{1-t / 0.71}\right)^{2}
$$

but their coupling $G_{\rho}(t)$ to the $\gamma \rho$ vertex is unknown. We find that a good description of the data is provided by the choice

$$
G_{\rho}(t)=\frac{1}{1-t / 0.71}
$$

Putting these things together, we have for the soft part of the amplitude for $\gamma p \rightarrow \rho p$

$$
T_{\mathrm{SOFT}}(s, t)=i F(t) G_{\rho}(t)\left[A_{P_{1}}\left(\alpha_{P_{1}}^{\prime} s\right)^{\alpha_{P_{1}}(t)-1} e^{-\frac{1}{2} i \pi\left(\alpha_{P_{1}}(t)-1\right)}+A_{R}\left(\alpha_{R}^{\prime} s\right)^{\alpha_{R}(t)-1} e^{-\frac{1}{2} i \pi\left(\alpha_{R}(t)-1\right)}\right]
$$

with

$$
A_{P_{1}}=6.0 \quad A_{R}=15.9
$$

The amplitude is normalised such that $d \sigma / d t=|T(s, t)|^{2}$ in $\mu b \mathrm{GeV}^{-2}$.

Figure 2 shows the differential cross section at $\sqrt{ } s=6.86$ and $10.4 \mathrm{GeV}$, together with CERN Omega data ${ }^{[2]}$. The data are not normalised. The solid line in figure 3 shows the same fit at $\sqrt{ } s=94 \mathrm{GeV}$, together with ZEUS data ${ }^{[1]}$ (which are normalised). The success of the fit at small $t$ is evidence that 

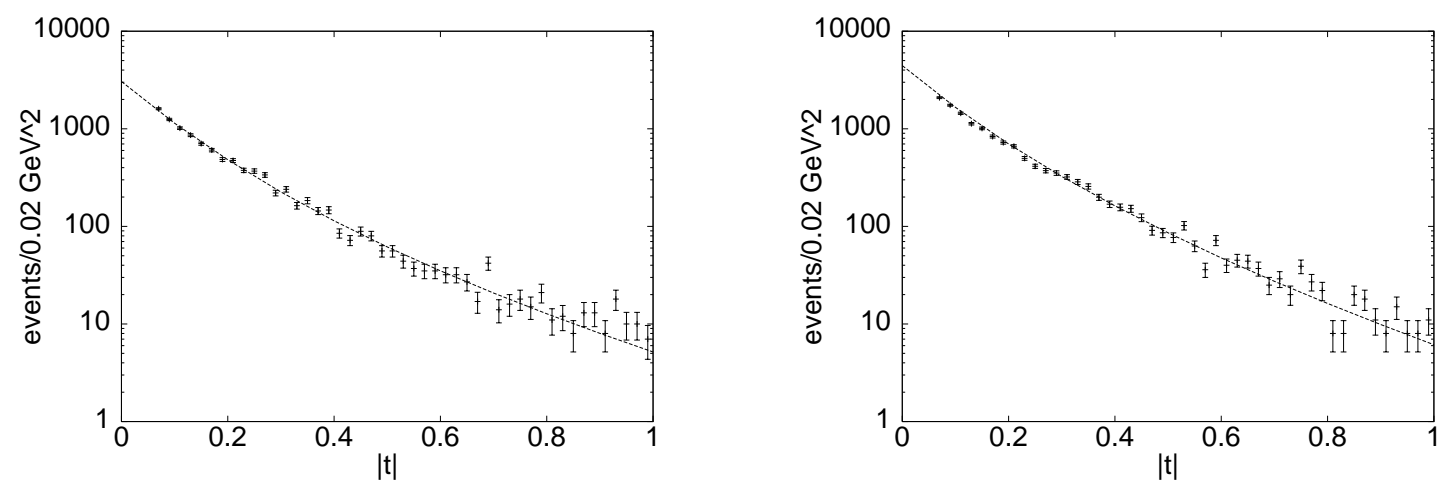

Figure 2: Data ${ }^{[2]}$ for $\gamma p \rightarrow \rho p$ at $\sqrt{ } s=6.86$ and $10.4 \mathrm{GeV}$, with Regge fit.

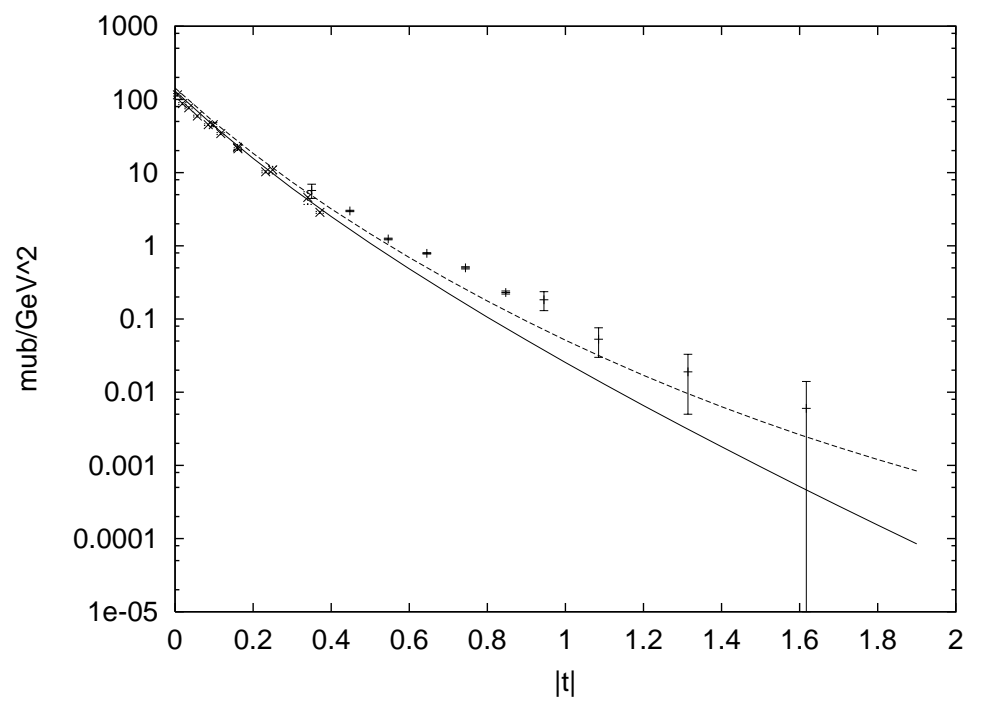

Figure 3: ZEUS data ${ }^{[1]}$ for $\gamma p \rightarrow \rho p$. The lower- $t$ data are at $\sqrt{ } s=71.7 \mathrm{GeV}$ and the higher- $t$ at $94 \mathrm{GeV}$. The solid line is the Regge fit with soft exchanges only; the dashed line includes the hard pomeron (the fits change very little between the two energies)

the classical value 0.25 of the soft-pomeron slope $\alpha_{P_{1}}^{\prime}$ is correct. The dashed lines in figures 1 and 3 include an additional contribution which we now discuss.

What we have said so far should be uncontroversial. The remainder of this paper concerns the hard pomeron and so may be less generally accepted, though it adds to the already-strong body of evidence in support of the concept. We first introduced ${ }^{[9]}$ a hard pomeron, with intercept $\alpha_{P_{0}}$ a little greater than 1.4, to explain the data for the proton structure function $F_{2}\left(x, Q^{2}\right)$ at small $x$. We then observed ${ }^{[3]}$ that the ZEUS data ${ }^{[10]}$ for the charm component $F_{2}^{c}\left(x, Q^{2}\right)$ of $F_{2}\left(x, Q^{2}\right)$ seem to confirm its existence, and tentatively deduced the slope of the trajectory from the H1 data ${ }^{[11]}$ for the differential cross section for the process $\gamma p \rightarrow J / \psi p$ :

$$
\alpha_{P_{0}}(t)=1.44+\alpha_{P_{0}}^{\prime} t \quad \alpha_{P_{0}}^{\prime}=0.1
$$

We found also that the coupling $G_{J / \psi}(t)$ to the $\gamma J / \psi$ vertex is rather flatter in $t$ than the coupling $G_{\rho}(t)$ to the $\gamma \rho$ vertex that we specify in $(3)$, and we took it to be constant. 

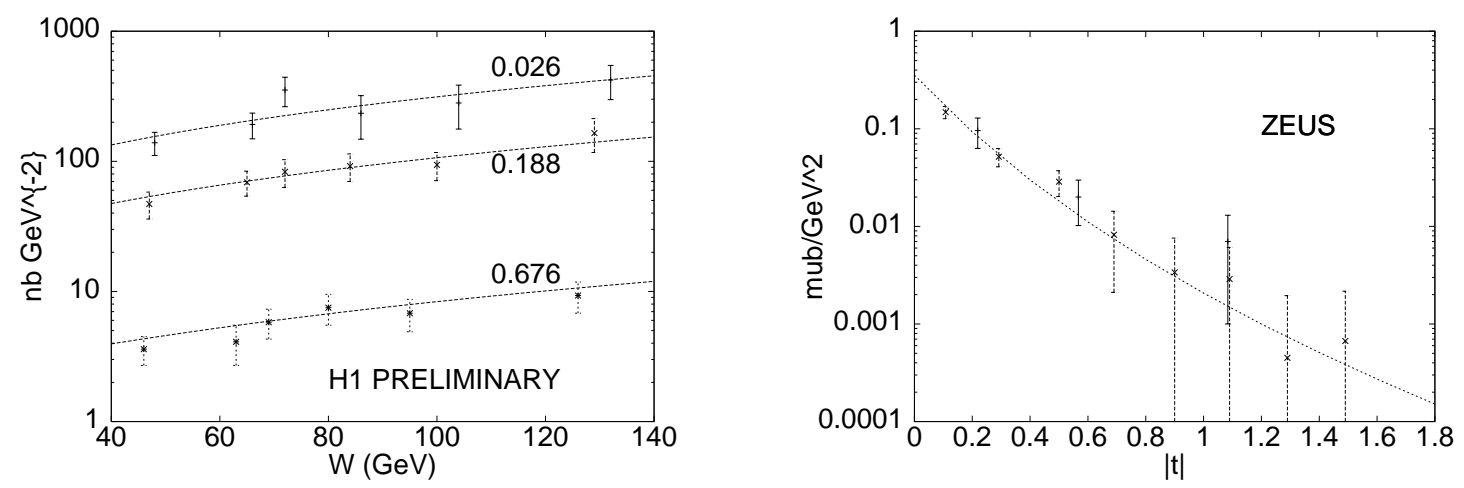

Figure 4: $\gamma p \rightarrow J / \psi p:$ H1 data ${ }^{[11]}$ at three $t$ values, and ZEUS data ${ }^{[1]}$ at $\sqrt{ } s=94 \mathrm{GeV}$. The fits include the hard and soft pomeron contributions

So the hard-pomeron contribution to the amplitude for $\gamma p \rightarrow J / \psi p$ is taken as

$$
i F(t)\left[A_{P_{0}}\left(\alpha_{P_{0}}^{\prime} s\right)^{\alpha_{P_{0}}(t)-1} e^{-\frac{1}{2} i \pi\left(\alpha_{P_{0}}(t)-1\right)}\right]
$$

This differs from what we used in reference [4] in that we divide $s$ by the mass scale $s_{0}=1 / \alpha_{P_{0}}^{\prime}$ before raising it to the Regge power. We assume Zweig's rule, so that the $f, a_{2}$ trajectory decouples and $A_{R}=0$, although a contribution from the soft pomeron is retained. Figure 4 shows the comparison with the $\mathrm{H} 1$ data $^{[11]}$ at three values of $t$, and the ZEUS data ${ }^{[1]}$ at $\sqrt{ } s=94 \mathrm{GeV}$, taking

$$
A_{P_{0}}=0.016 \quad A_{P_{1}}=0.17
$$

Our fits to the data for $F_{2}$ at small $x$ and for $F_{2}^{c}$ suggest that the coupling of the pomeron to quarks is flavour-blind. So, in order to relate the strengths of the hard-pomeron couplings in the processes $\gamma p \rightarrow J / \psi p$ and $\gamma p \rightarrow \rho p$ we need just to include wave-function effects. Although the hard pomeron couples to photon-induced reactions, its coupling to purely hadronic processes is extremely small ${ }^{[9]}$. So it seems reasonable to assume that it is the pointlike component of the photon that is largely responsible, rather than the hadron-like component. This in turn implies that in $\gamma p \rightarrow V p$, the strength of the hard-pomeron coupling depends on the magnitude of the $V$ wave function at the origin and the relevant quark charges, and that therefore it is proportional to $\sqrt{\Gamma_{V \rightarrow e^{+} e^{-}} / m_{V}}$. This implies that for $\gamma p \rightarrow \rho p$ we should use

$$
A_{P_{0}}=0.036
$$

Adding such a hard-pomeron term to the amplitude gives the dashed curve in figure 3 .

It might have been thought that a Regge cut, for example from two-pomeron exchange, could have been used to explain the $\rho$ data at larger $|t|$ as the cut has a less strong t-dependence than the pole. However the cut has the opposite sign to the pole, so far from enhancing the cross section at large $|t|$ it actually reduces it.

Finally we apply the model to $\phi$ photoproduction. As before we can use the flavour-blind nature of the coupling of the hard pomeron to quarks to specify its contribution uniquely. This gives

$$
A_{P_{0}}=0.014
$$

Just as for the $\rho$ there are two unknowns in the soft pomeron contribution to $\phi$ photoproduction: the magnitude of the coupling of the soft pomeron to strange quarks and the mass scale in the $\phi$ form 
factor. We know that Vector Meson Dominance is not a good approximation for the $\phi$ and that wave function effects are important $[4]$, so the normalisation can only be specified by the data. Naively the mass in the form factor is simply that of the $\phi$ but higher-mass $s \bar{s}$ states must contribute making the effective mass somewhat larger. In analogy with the $\rho$ case we use

$$
G_{\phi}=\frac{1}{1-t / 1.5}
$$

choosing 1.5 instead of 0.71 on the grounds that $m_{\phi}^{2} \sim 2 m_{\rho}^{2}$. As for the $J / \psi$ we can neglect any contribution from $f, a_{2}$ exchange. Fitting the soft pomeron coupling to the data yields

$$
A_{P_{1}}=1.49
$$

and the results are shown in figure 5 .
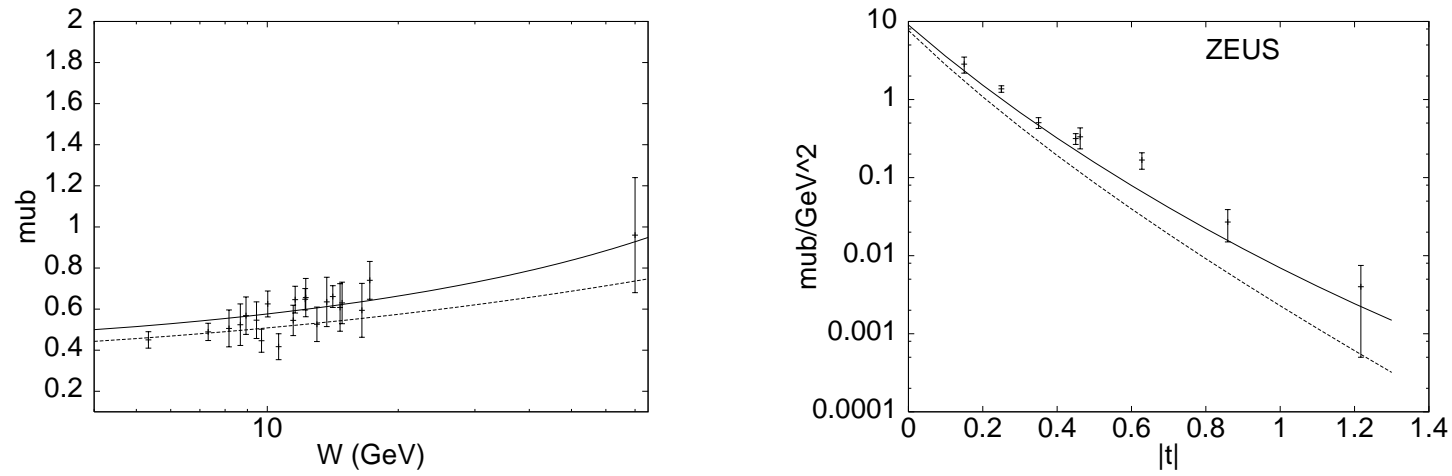

Figure 5: $\gamma p \rightarrow \phi p:$ Data for the total cross section ${ }^{[12]}$ and the differential cross section ${ }^{[1][13]}$ at $\sqrt{ } s=94 \mathrm{GeV}$. The dashed lines show the soft pomeron contributions and the solid lines include also the hard pomeron

This research is supported in part by the EU Programme "Training and Mobility of Researchers", Networks "Hadronic Physics with High Energy Electromagnetic Probes" (contract FMRX-CT96-0008) and "Quantum Chromodynamics and the Deep Structure of Elementary Particles" (contract FMRX-CT98-0194), and by PPARC

\section{References}

1 ZEUS collaboration: J Breitweg et al, Europ Phys Jour C1 (1998) 81 and hep-ex/9910038

2 D Aston et al, Nuclear Physics B209 (1982) 56

3 A Donnachie and P V Landshoff, hep-ph/9910262

4 A Donnachie and P V Landshoff, Physics Letters B348 (1995) 213

5 A Donnachie and P V Landshoff, Physics Letters B296 (1992) 227

6 G Gounaris and J J Sakurai, Physical Review Letters 21 (1968) 244

F M Renard, Nuclear Physics B15 (1970) 267

7 G Veneziano, Nuovo Cimento 57A (1968) 190 
8 A Donnachie and P V Landshoff, Nuclear Physics B231 (1983) 189

9 A Donnachie and P V Landshoff, Physics Letters B437 (1998) 408

10 ZEUS collaboration: A Breitweg et al, hep-ex/9908012

11 H1 Collaboration, submitted to the International Europhysics Conference on High Energy Physics HEP99, Tampere, Finland, July 1999

12 J Busenitz et al, Physical Review D40 (1989) 1

13 ZEUS collaboration: M Derrick et al, Physics Letters B377 (1996) 259 